\title{
Agile Manufacturing - An Overview
}

\author{
Manivelmuralidaran. V \\ Department of Mechanical Engineering, Kumaraguru College of Technology, \\ Coimbatore-641049, India
}

\begin{abstract}
Smaller lot sizes, shorter times to market, and lower manufacturing costs are typical requirements of a modern manufacturing facility. Under such constraints, agile manufacturing systems are desirable. Agile manufacturing uses technology, organizational approaches, systems and people in a manufacturing concept which truly belongs to the $21^{\text {st }}$ century and which transcends existing methodology to facilitate ability of the organization to react to rapid change and respond to an increasingly unpredictable marketplace. Agile manufacturing can produce what customers want, when and exactly how they want it, at a costeffective price. The main aim of this paper is to presents the various possible research domains in the development of agile manufacturing era. The objective of this paper is to brief an overall idea and research needs of agile manufacturing with the focus of current fluctuations in the market scenario.
\end{abstract}

Keywords Agile Manufacturing, Lean manufacturing, Manufacturing Engineering, Production planning and control

\section{INTRODUCTION}

World-class performance is a moving target that requires constant attention, effort and the process is a never ending journey. In the past, economies of scale ruled the manufacturing world and everybody knew that mass production and full utilization of plant capacity was the way to make money. This style of manufacturing resulted in inflexible plants that could not be easily reconfigured, and were associated with swollen raw materials, work-in-process and finished goods inventories. For the elimination of excess inventory, shortened lead-times, requirement of flexibility in the flow lines and to enhance the advanced levels of quality in both products and customer service, industry analysts have popularized the terms 'world-class manufacturing' and agile manufacturing.

\section{AGILE MANUFACTURING}

Facing the competitive market, industrial manufacturers are hard pressed to adopt novel strategies and technologies to enhance product quality, to cut manufacturing cost and to reduce product lead time. Agile Manufacturing is primarily a business concept. Its aim is quite simple - to put our enterprises way out in front of our primary competitors. In Agile Manufacturing, our aim is to combine our organization, people and technology into an integrated and coordinated whole. Agile manufacturing can be defined as the capability to survive and prosper in a competitive environment of continuous and unpredictable change by reacting quickly and effectively to changing markets, driven by customer-designed products and services.

The concept of agility refers to a business model which makes an organization immune to damage caused by unpredictable events and changing circumstances. This is achieved by the organization being able to react and adapt rapidly [5]. The coming surprises of the future will not make an agile enterprise vulnerable because that enterprise will have already put in place a set of business practices, organization-wide culture changes and technology which will allow it to be prepared for any change in circumstances, market forces or customer needs. Agile Manufacturing is a combination of speed and flexibility that is difficult to achieve because it requires radical changes to traditional thinking.

The concept of Agile Manufacturing is built around the synthesis of a number of enterprises that each have some core skills or competencies which they bring to a joint venturing operation, which is based on using each partner's facilities and resources [7]. Central to the ability to form these joint ventures is the deployment of advanced information technologies and the development of highly nimble organizational structures to support highly skilled, knowledgeable and empowered people. Agile Manufacturing builds on what is good in lean manufacturing and uses what can be adapted to western cultures, but it also adds the power of the individual and the opportunities afforded by new technologies.

Agile Manufacturing enterprises will be capable of rapidly responding to changes in customer demand. They will be able to take advantage of the windows of opportunities that, from time to time, appear in the market place. With Agile Manufacturing it is possible to develop new ways of interacting with customers and suppliers. The customers will not only be able to gain access to the products and services, but will also be able to easily assess and exploit the competencies, so enabling them to use these competencies to achieve the things that they are seeking.

The goal of this paper is to review several different aspects of agile manufacturing (AM) and to identify key aspects of agile manufacturing and outlook for the future of agile manufacturing technologies.

\subsection{Comparison of Agile manufacturing and other manufacturing methods:}

In traditional manufacturing system the long cycle time is with the high inventory which causes delay in the goods delivery. The problem here is the lack of strategy and no employee involvement and financial secrecy and record inaccuracy. In the context of world class manufacturing the advantages are long term profitability, productivity 
improvement and less time to market in contrast to the traditional manufacturing system. The agile manufacturing system focusing in the integration of design and manufacturing and every aspect of manufacturing under one roof.

In Contrast to the traditional manufacturing strategy, the agile manufacturing concentrates on the customer enrichment, competitiveness through co-operation and this could be achieved by integration of the people, information and technology on a same roof. This could also be achieved by highly educated and trained workforce. Enhancing the competitiveness among the competitors and built the cooperation from all the enterprises and thus influence the knowledge sharing and sharing the technological innovations.

\subsection{Needs of agile manufacturing system}

The key to agility however, lies in several places. An agile enterprise needs highly skilled and knowledgeable people who are flexible, motivated and responsive to change. An agile enterprise also needs new forms of organizational structures which engender non-hierarchical management styles and also stimulate and support individuals as well as cooperation and team working. Agile manufacturing enterprises also need advanced computer based technologies to integrate information and to share the knowledge base [2].

To achieve Agile Manufacturing, enterprises will have to bring together a wide range of knowledge in the design of a manufacturing system that encompass suppliers, customers. It should also addresses all dimensions of the system including organisation, people, technology, management accounting practices, etc. Most importantly the inter-related nature of all these areas needs to be recognised and an interdisciplinary manufacturing systems design method adopted as standard practice[3]. This means going beyond the multidisciplinary approaches that are currently being adopted and looking at areas between professions.

\subsection{Challenges of Agile manufacturing} Interdisciplinary design will form the basis of designing Agile Manufacturing systems in the new knowledge intensive era. Interdisciplinary design however, means more than just applying knowledge from other domains, such as psychology and organisational science, to the design of Agile Manufacturing systems. It also implies looking into the unexplored areas between these disciplines and the areas where they overlap, to find a new insights, new knowledge, new and original solutions. This is one of the most important challenges that managers and system designers and integrators will face in the years ahead. The interdisciplinary design leads us to new approaches and new ways of working and of thinking [4]. However, to successfully adopt an interdisciplinary design method, we also need to:

- Challenge the present design strategies and develop new and better approaches;

- Question the established and cherished beliefs and theories, and develop new ones to replace those that no longer have any validity;

- Consider how to address organisation, people and technology, and other issues in the design of manufacturing systems, so that the systems can achieve the better performance for the agile manufacturing environment and for the people who form a part of this system.
- Make use of the innovative technology in a way that makes human skill, knowledge, and intelligence more effective and productive which allows industries to tap into the creativity and talent of all people in an enterprise.

The challenges that face with respect to all these issues are enormous. In the world of manufacturing the challenges and problems are very complex. There are a massive number of interconnections between the various components and elements. A manufacturing enterprise is so complex that, in the past, it has been impossible to cope with it as a whole, and it has been necessary to reduce it into manageable areas which have tended to be examined separately.

\subsection{Agile Manufacturing world wide scenario}

The Agile Manufacturing Enterprise Forum (AMEF) at the Iacocca Institute of Lehigh University was created to disseminate the ideas of agile manufacturing and to increase the pace and scope of the transition to an agile manufacturing industry. The Agile Manufacturing Initiative aims to develop, demonstrate, and evaluate the advanced design, manufacturing and business transaction processes in the agile environment. The Concurrent Technologies Corp. (CTC) is developing an agile manufacturing testbed to provide Department of Defense (DoD) with increased weapon system readiness and added system mobility. The Agile Aerospace Manufacturing Research Center (AAMRC) at the University of Texas at Arlington is conducting research on agile business practices, business process identification and characterization, and enabling technologies. The Manufacturing Research, Education and Outreach Program at the University of Illinois at Urbana-Champaign is developing computer integrated manufacturing and machine tool systems. The Electronic Agile Manufacturing Research Institute (EAMRI) at Rensselaer Polytechnic Institute (RPI) is focusing on electronics product realization in distributed manufacturing environments using improved information infrastructures and architectures.

Agile manufacturing makes use of modern information technology to form virtual enterprises, which agilely respond to the changing market demands. A virtual enterprise, different from a traditional enterprise, is constructed by partners from different companies, who collaborate with each other to design and manufacture high quality and customized products. It is product-oriented, team-collaboration styled, and featured as fast and flexible. Frequent and dynamic interactions among partners in agile manufacturing enterprises entail the crucial role of an Agile Manufacturing Information System (AMIS). It is up to the AMIS to provide partners with integrated and consistent information, as well as to manage partner transactions accessing the information.

\subsection{Transition to Agile Manufacturing}

All the relevant dimensions of Agile Manufacturing, such as organisation, people, technology, management accounting, etc. are however, all written in different books and taught by different people. To make the transition to Agile Manufacturing we need to:

- Examine and define the underlying conceptual framework on which Agile Manufacturing enterprises will be built.

- Explore and understand the nature of the mass production paradigm and the nature of the cultural 
and methodological difficulties involved in the transition to Agile Manufacturing.

- Define a methodology for designing a $21^{\text {st }}$ century manufacturing enterprise.

The agile manufacturing is based on a systems perspective of technology, organisation and people, tied to clear business vision and goals. This will help the researchers to understand the full complexity of designing a $21^{\text {st }}$ century manufacturing enterprise that is agile manufacturing system.

\subsection{Characteristics of Agile Manufacturing}

The characteristics of agile manufacturing are at the strategic level where strategic dimensions of agile manufacturing are identified as follows: 1. Enriching the customer - an agile company is one that is perceived by its customers as enriching them in a significant way, not only itself

2. Cooperating to enhance competitiveness - cooperation internally and with other companies is an agile competitor's operational strategy of first choice 3. Organizing to master change and uncertainty - an agile company is organized in such a way as to allow it to thrive on change and uncertainty, its structure is flexible enough to allow rapid configuration of human and physical resources; 4. Leveraging the impact of people and information - an agile company's management nurtures an entrepreneurial company culture that leverages the impact of people and information on operations. From these strategic dimensions of agility, one can move to consider the tactical and technological dimensions. In this effort, the AMRIs are concerned with research and development efforts to better understand the enabling technologies for agile manufacturing.

The benefits of Agile Manufacturing are Short time-tomarket, fast new product development, Short/fast order processing, Low volumes, Low quantities, High product mix, Configurable components, Fast supplier deliveries, Short lead times, Short cycle times, Highly flexible and responsive processes, Highly flexible machines and equipment, Use of advanced CAD/CAM, Quick changeover, Empowered employees.

\section{POTENTIAL RESEARCH PROSPECTS IN AGILE MANUFACTURING}

There are lot of issues are related with the manufacturing and production in agile manufacturing environment. Some of the potential area are Product and manufacturing systems design, Process planning [5], Production planning scheduling and control, Facilities design, Facilities location, Material handling and storage systems, Integrated information systems, Information systems designed for supporting specific areas of Information exchange, Supply chain Strategies, Partner selection [4,6], Human factors.

\subsection{Human Factors in Agile Manufacturing}

In manufacturing we have tended to treat organisation, people and technology issues independently, and for the most part this division of knowledge has worked well in the past. In a report of Human Factors in Agile Manufacturing by Chris Forsythe at Sandia National Laboratories pointed out that Agile manufacturing will precede, by raising human factors issues and applying the knowledge and skills gained from other domains [1]. There is an opportunity for human factors to assume an important role positively influencing the future of agile manufacturing. Still Agile manufacturing poses many questions best answered by human factors. Our willingness, as a researcher is to address these questions and enhance the agile manufacturing environment.

\subsection{Production planning, scheduling and control}

Production planning, scheduling and control is concerned with manufacturing the right product types, in the right quantities, at the right time, at minimum cost and meeting quality standards. Production planning, scheduling and control are the heart of manufacturing firms [7]. He and Kusiak (1996) developed two models for production planning and scheduling in a virtual corporation[7]. This paper considers that every virtual corporation is formed by a number of manufacturing companies (manufacturing centres) and assembly companies (assembly centres). The first model allocates products to the assembly centres so that the total average set-up and inventory cost is minimized. The model is solved with an efficient heuristic algorithm. The second model deals with scheduling the manufacturing centres. In this paper, the authors assume that each firm has a direct production capability (each one can produce di. erent products). Additionally, they assume that partners are manufacturing centres or assembly centres, but not both.

Don-Taylor (1996) introduces the Design for Global Manufacturing and Assembly (DFGMA) as a tool to assist designers in making optimal sourcing, capital procurement, and market timing decisions in a multi-facility, global environment. More specifically, DFGMA is a mathematical tool that enables production planners optimally to introduce a new product into a suitable production line. The first of the new product with the existing mix is considered and the dynamic nature of the product mix is also considered [2]. DFGMA can also be used as a tool for performing sensitivity analysis for various costs, productivity levels, product configuration, mix assumptions at each facility, or analysis of parameters of interest to design, marketing and manufacturing. The mathematical model was formulated as a mixed-integer linear program (MILP) where the objective function specifies the minimization of all costs associated with the design, manufacture, inventory, transportation, logistics, set-up, tooling and capital procurement, to support the introduction of a new product. Six different types of constraints were included in the model. The LINDO software package was used to solve the model. Further work can be done in evaluating the use of the DFGMA model for concurrent sourcing decisions of multiple new products to sites characterized by various existing mixes. In addition DFGMA should be tested in situations with more explicit model market timing issues. Another area of research interest is the applicability and evaluation in a real system.

Sarmiento et al. (1999) reviewed recent work in the area of integrated analysis on systems that explicitly consider logistics (transportation systems) integrated into other functions in production, and also they identified areas where further research is needed. In addition, the authors were interested in identifying work done at the strategic or tactical level [8]. Among the conclusions, the authors' state that the integration of the logistics function into the analysis of previously isolated production is also an important criteria.

L. M. Sanchez et al. work has the potential of providing significant benefits to companies, in the form of cost savings and efficiency improvement. One problem encountered in forming a virtual corporation is to assign tasks to each partner 
and to schedule the production based on the capabilities of each one of them [8]. They remark that it is important to have an integrated production planning, scheduling and control system. Still lot more areas are to be research for the agile manufacturing environment.

Another important aspect of agile manufacturing is the focus towards the customer feedback and replies. Though less amount of paper work has been done in this aspect and lot more have to be explored to enhance the quick response to the customer feedback. So it is essential to plot a road map to the processes and to speed up the procedure to quickly response to the customer needs. The research in this direction is very limited and this could be taken as a starting point for the exploration of agile manufacturing environment.

The key points to understand in the agile manufacturing are it is a strategy aimed at developing capabilities (the enterprise platform) to prosper in the 21 st century. In this respect it is similar to a manufacturing strategy in that it should support business and marketing strategies. However, these strategies also need to be modified to take advantage of agile manufacturing capabilities. As a strategy, agile manufacturing is concerned with objectives, structures, processes and resources and not with individual point solutions, particular technologies, methods, etc. considered in isolation [9]. The emphasis is on designing the enterprise as a whole so that certain characteristics are achieved and not on the piecemeal adoption of quick fixes, prescriptions and panaceas. Agile manufacturing may require some current best practices, lean production concepts, technologies and taken-for-granted assumptions to be re-evaluated, modified or even abandoned. In the same way that mass-production marginalised many craft-based firms, agile manufacturing is likely to marginalise many mass production firms, even those with lean production enhancements. One of the biggest problems to overcome is the misunderstandings that lean and agile are synonymous. They are not, although most of what is portrayed as agile is in fact lean.

There is a need to view the agile manufacturing concept into the service industries and a quality management system should be formulated for assurance of quality in agile manufacturing enterprise and a well cost accounting method is to be established and designed to measure the cost of a product and decide about the product mix in the agile manufacturing environment[3].

\section{CONCLUSION}

Agility as a subject is still a developing area. Companies are starting to move towards agile behavior, often not in a planned way, more usually they "fall into it" and as a result it tends to be operational and lacks strategic direction. As a first step, companies need to understand the basic ideas and become intelligent respondents to the concepts. Agility is a long term issue for businesses and achieving agility is a journey, not an objective to be attained before moving on to something else. The implementation of agility is still very much a frontier activity, involving radically new concepts concerning strategies, organisation, people and technologies. It takes businesses into a domain where fundamental and taken for granted assumptions are challenged. Agility is a paradigm shift and before one can move forwards one has to understand the existing paradigm and to face up to the often painful task of accepting that current practices and beliefs are no longer appropriate or relevant.

\section{REFERENCES}

[1] Chris Forsythe., Ashby.M.R., Human factors in agile manufacturing, Journal of Ergonomics in Design, 4(1), pp. 15-21Cho.H., Jung.M., Kim.M., Enabling Technologies of the agile manufacturing and its related activities in Korea, Computers and Industrial Engineering Journal, 30(3),pp. 323-324

[2] Don-Taylor,G. and Nagi,R., Agile manufacturing in material handling and logistics:Implications and a research agenda. Progress in Material Handling Research, 1996, pp. $9 \pm 13$.

[3] Gunasekaran.A, Agile manufacturing :endblers and an implementation frame work, International Journal of Production Research, Vol 36, No.5,1223-1247.

[4] Gupta.P., Nagi.R, Flexible optimization framework for partner selection in agile manufacturing. IERC Proceedings 1995, 4th Annual Industrial Engineering Research Conference, Norcross, GA, USA, pp 691-700

[5] Gupta.S, Herrmann.J.W., Lam.G, Minis.I, Automated high level process planning to aid design for agile manufacturing, IERC Proceedings 1997, 6th Annual Industrial Engineering Research Conference, Norcross, GA, USA, pp1-19

[6] Gupta.P.,Nagi.R., Process partner selection in agile manufacturing using linguistic decision making. Department of Industrial Engineering, State University of Newyork

[7] He.D, Kusiak.A., Design for Agility: A Scheduling Perspective, Robotics and Computer-Integrated Manufacturing Systems, Vol. 14, No. 4, 1998, pp. 415427.

[8] Luis M. Sanchez ;Rakesh Nagi, A review of agile manufacturing systems, International Journal of Production Research, 39(16), November 2001 , pp 3561 3600

[9] Wang.Z.Y.,Rajkumar.K.P., Kapoor.A., Architecture for agile manufacturing and its interface with computer integrated manufacturing, Journal of Materials Processing and Technology, 61(1), 1996, pp. 99-103 